\title{
Lipoma of conus medullaris without spinal dysraphism in an adult
}

Sir,
A 46 -year-old male was admitted with a two-year
history of low back pain, followed by radiating
pain in the lateral aspect of left upper thigh. At
admission, neurological examination showed motor
weakness of grade $4 / 5$ in both the lower limbs,
hypoesthesia in the lateral aspect of left upper thigh
in the L1-2 dermatomes., normal tendon reflexes and
no pathological reflexes. Magnetic resonance imaging
(MRI) of lumbar spine revealed an intradural ovoid
mass of approximately $13 \times 15 \times 43 \mathrm{~mm}^{3}$ in size
occupying T11 to L1, which was hyperintense on both
T1-weighted and T2-weighted images [Figure 1 a and b].
He underwent T11 to L1 laminectomy. At operation,
the tumor removal was subtotal as there was no 


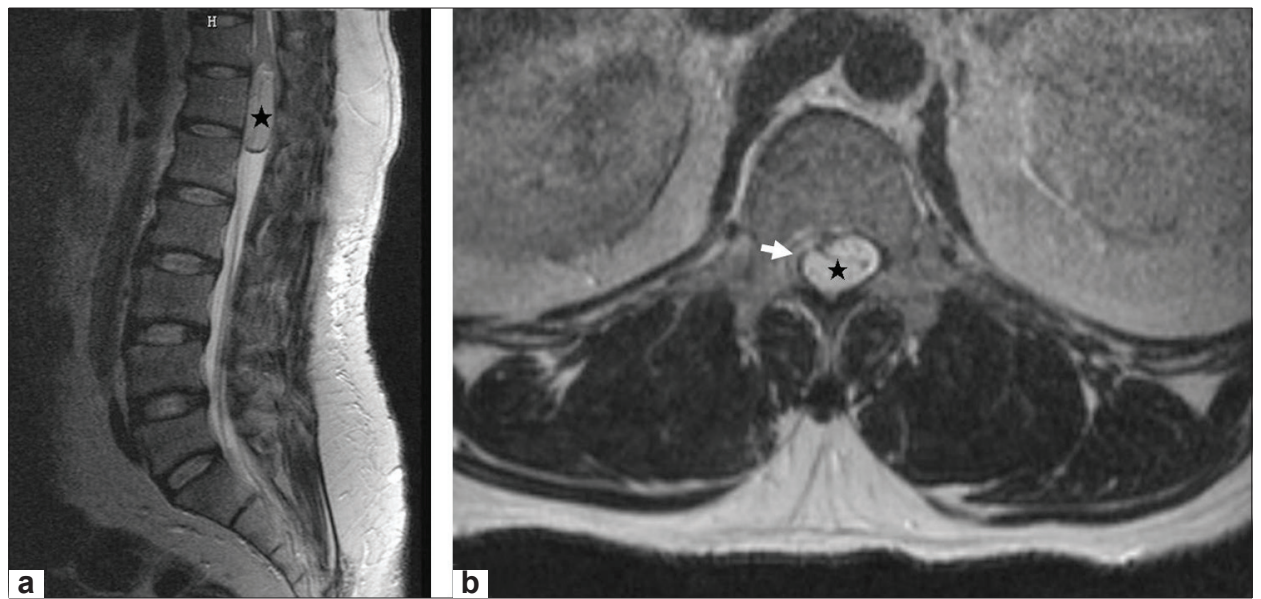

Figure 1: (a) Sagittal view of T2-weighted magnetic resonance images revealing the intraspinal mass (asterisk) in the T11 to L1 level, which was connected to the conus medullaris; (b) Axial view of T2-weighted magnetic resonance images revealing the intradural spinal tumor (asterisk), compressing the conus medullaris (white arrow) severely

obvious interface between the tumor, cord, and nerve roots. The pathological examinations confirmed the diagnosis of lipoma. Postoperatively motor weakness worsened and developed loss of anal tone, and urinary incontinence. The incontinence improved one month after the operation. Motor power in both the lower limbs improved from grade 2 to grade 4 after six months of rehabilitation.

Lipoma of conus medullaris without spinal dysraphism in an adult is a rare entity and only seven cases have been reported in the English literature till 2002. ${ }^{[1]}$ Many causes such as proliferation of adipose cells, deposition of fat in the connective tissue, metaplastic differentiation of persisting embryonic meninges, and abnormal embryologic development (dysraphism) have been hypothesized for the development of these tumors. ${ }^{[2]}$ The clinical presentations of intradural spinal lipoma are mainly related to the mass effect. ${ }^{[3]}$ Most of these patients have the symptoms for more than twoyears before the diagnosis. MRI is the best diagnostic modality to evaluate spinal lipoma and delineate the adjacent neural structures. The fat component can be easily confirmed by using the fat-suppression images. Surgical intervention is indicated for the patients with progressive neurological symptoms. The goal of surgery is not only to remove the tumor, but also to preserve the neurological functions. With improvements in the neurosurgical techniques, use of carbon dioxide laser has been shown to have surgical precision, and maximal removal of the tumor with minimal surrounding trauma and improved hemostasis. Use of carbon dioxide has less electrical interference with the intraoperative evoked potential recordings. However, as there is often no clear-cut margin between lipoma and the cord, aggressive total removal of tumor is hard to achieve and is also not recommended. Partial surgical excision with wide laminectomy, laminoplasty or duroplasty also has been advocated to treat the intraspinal lipoma. ${ }^{[4]}$ The management of lipoma of conus medullaris without spinal dysraphism remains a challenge.

Cheng-Ta Hsieh, Jui-Ming Sun ${ }^{1}$, Ming-Ying Liu Department of Neurological Surgery, Tri-Service General Hospital, National Defense Medical Center, ${ }^{1}$ Surgery, Songshan Armed Forces General Hospital, Taipei, Taiwan, Republic of China. E-mail:nogor@mail2000.com.tw

\section{References}

DOI: $10.4103 / 0028-3886.59497$

1. El Mostarchid B, Ali A, Maftah M, Mansouri A, Laghzioui J, Kadiri B, et al. Non-dysraphic intramedullary spinal cord lipoma. A case report. Joint Bone Spine 2002;69:511-4.

2. Kasliwal MK, Mahapatra AK. Surgery for spinal cord lipomas. Indian J Pediatr 2007;74:357-62.

3. Koyanagi I, Hida K, Iwasaki Y, Isu T, Yoshino M, Murakami T, et al. Radiological findings and clinical course of conus lipoma: Implications for surgical treatment. Neurosurgery 2008;63:546-51; discussion 51-2.

4. Bekar A, Sahin S, Taskapiloglu O, Aksoy K, Tolunay S. Intradural spinal lipoma: Report of a thoracic case and a lumbar case. Turkish Neurosurgery 2004;14:52-6.

Accepted on 06-08-2009 